\title{
DISTRIBUCIÓN Y CARACTERIZACIÓN MORFOLÓGICA DE HYCLEUS DUODECIMMACULATUS (OLIVIER, 1811) (COLEOPTERA, MELOIDAE), UNA ESPECIE POCO CONOCIDA DEL OESTE DE MARRUECOS
}

\author{
José L. Ruiz* \& Mario García-París**;† \\ * Instituto de Estudios Ceuties. Paseo del Revellin, 30. 51001 Ceuta. España. \\ E-mail: euserica@hotmail.com \\ ** Museo Nacional de Ciencias Naturales. C.S.I.C. c/ José Gutiérrez Abascal, 2. 28006 Madrid. España. \\ E-mail: mparis@mncn.csic.es \\ urn:Isid:zoobank.org:author:5FD2079F-52BF-41F4-8C88-70593E306DA0 \\ ${ }^{+}$autor para correspondencia
}

\section{RESUMEN}

Hycleus duodecimmaculatus (Coleoptera, Meloidae, Mylabrini) es un endemismo de Marruecos muy poco conocido, cuya distribución se extiende por la franja costera-subcostera del Marruecos atlántico, entre Larache y la región de Tiznit (Arbaa Ait Ahmed). En este trabajo, realizado principalmente a partir del estudio de más de un centenar de ejemplares conservados en su mayor parte en la colección del Museo Nacional de Ciencias Naturales (MNCN-CSIC, España), se aportan nuevas citas geográficas incluyendo el Rif occidental, y se confirma su presencia en Marrakech (falda noroccidental del Alto Atlas). A la vista de la escasez de información existente para esta especie, dispersa y a veces errónea, se sintetiza la información corológica disponible, y se ofrecen datos sobre fenología de los adultos y tipología general de los hábitats que ocupa. Desde el punto de vista taxonómico, se redefinen los caracteres diagnósticos de la especie y se ilustran diversos aspectos de la variabilidad morfológica intra e interpoblacional.

Palabras clave: Noroeste de África; Marruecos; taxonomía; distribución geográfica; variabilidad morfológica; autoecología; Hycleus duodecimmaculatus.

\section{ABSTRACT}

Distribution and morphological characterization of Hycleus duodecimmaculatus (Olivier, 1811) (Coleoptera, Meloidae), a poorly known species from western Morocco

Hycleus duodecimmaculatus (Coleoptera, Meloidae, Mylabrini) is a poorly known species endemic to Morocco. Its geographic range extends along the coastal-subcoastal fringe of the Atlantic Coast, between Larache and Tiznit region (Arbaa Ait Ahmed). In this paper we study more than a hundred specimens, mostly preserved in the collection of the Museo Nacional de Ciencias Naturales (MNCN-CSIC, Spain). We provide new geographic records, including the Western Rif and confirm its presence in Marrakech (northwestern slopes of the High Atlas). Because of the scarcity of information, disperse and sometimes erroneous, we synthesize information available on chorology and phenology, and characterize the typology of the species' habitat. From a taxonomic point of view, we redefine the species' diagnostic characters and illustrate various aspects of the intra- and interpopulational morphological variability.

Key words: Northwestern Africa; Morocco taxonomy; geographic distribution; morphological variability; autoecology; Hycleus duodecimmaculatus.

Recibido/Received: 3/03/2014; Aceptado/Accepted: 2/06/2014; Publicado en línea/Published online: 22/08/2014

Como citar este artículo/Citation: Ruiz, J. L. \& García-París, M., 2014. Distribución y caracterización morfológica de Hycleus duodecimmaculatus (Olivier, 1811) (Coleoptera, Meloidae), una especie poco conocida del oeste de Marruecos. Grael/sia, 70(1): e006. http://dx.doi.org/10.3989/graellsia.2014.v70.110.

Copyright: (c) 2014 SAM y CSIC. Salvo indicación contraria, todos los contenidos de la edición electrónica de Graellsia se distribuyen bajo licencia de uso y distribución Creative Commons Reconocimiento no Comercial 3.0. España (cc-by-nc). 


\section{Introducción}

La tribu Mylabrini, ampliamente distribuida por el viejo mundo (Bologna \& Pinto, 2002), se encuentra representada en Marruecos por 5 géneros, Actenodia Laporte, 1840, Ceroctis Marseul, 1840, Croscherichia Pardo Alcaide, 1850, Hycleus Latreille, 1817 y Mylabris Fabricius, 1775, que agrupan a un total de 52 especies (Bologna, 2008; Ruiz \& García-París, 2008). Marruecos muestra, con diferencia, la mayor diversidad específica de Mylabrini del mediterráneo occidental (seguido de Argelia, con 46 especies), entre la que además destaca un componente endémico elevado $(21.1 \%, 11$ especies; datos extraídos de Bologna, 2008 y Ruiz \& García-París, 2008) en relación a otros países de su entorno geográfico.

El género Hycleus, de amplia extensión por las regiones etiópica, paleártica y oriental (Bologna \& Pinto, 2002), cuenta con 17 especies en territorio marroquí, de las cuales casi un tercio (29.4\%, 5 especies) son endémicas del país, de manera que integra el mayor porcentaje de endemismos, seguido de Mylabris $(26 \%, 6$ especies de un total de 23) (véase Bologna, 2008; Ruiz \& García-París, 2008). En general, las especies de Mylabrini exclusivas de Marruecos son muy poco conocidas, incluso en lo concerniente a aspectos básicos, tales como variabilidad morfológica, alfa-taxonomía, distribución geográfica y autoecología (e.g. Pardo Alcaide, 1954a, 1954b; Ruiz, 2000, 2004; García-París \& Ruiz, 2011), a pesar del interés patrimonial y de la importancia conservacionista de estos singulares elementos faunísticos.

Entre las especies de Hycleus exclusivas de Marruecos, se encuentra $H$. duodecimmaculatus (Olivier, 1811), que junto a los también endémicos Hycleus ruficornis (Fabricius, 1798) e Hycleus rufipalpis (Martínez de la Escalera, 1909) ${ }^{1}$, conforma un pequeño grupo de especies morfológicamente afines cuya área de distribución se limita a la franja costera y subcostera atlántica del país, con penetraciones hacia el interior (e.g. Martínez de la Escalera, 1909, 1914; Pardo Alcaide, 1954a, 1954b; Kocher, 1956a, 1964, 1969; Bologna, 2008). Aunque descrita hace algo más de dos siglos sobre material procedente de "Barbarie" (Olivier, 1811: 98-99, $\mathrm{n}^{\circ}$ 41, sub Mylabris duodecimmaculata), la información disponible sobre $H$. duodecimmaculatus es escasísima y se limita a contados

\footnotetext{
'Bologna (1994a) señaló a H. rufipalpis de las Islas Canarias a partir de un ejemplar etiquetado "Iles Canaries, 1897, C. Buchet leg., 100" (Muséum National d'Historie Naturelle, París), e indicó la necesidad de confirmar este registro y la presencia de una población estable de la especie en las Canarias. Machado \& Oromí (2000) la excluyen de la fauna canaria al considerarla (sic) "probable error de localidad o importación fortuita (1 ex., 1897)". Aunque Bologna (2008) mantiene dicha localización, estimamos que debe considerarse ajena a la fauna canaria, al igual que Machado \& Oromí (2000) y, por tanto, sería por el momento exclusiva de la región atlántica de Marruecos.
}

registros precisos y a observaciones puntuales de actividad de los adultos (véase "Resultados"), al igual que ocurre en las otras dos especies señaladas. En el mismo sentido, apenas se ha aludido en la literatura a los rangos de variabilidad de determinados caracteres morfológicos de cierta importancia diagnóstica (e.g. Pardo Alcaide, 1954a).

En el presente trabajo, se aportan nuevas citas de esta especie, y se señala por primera vez del Rif occidental. Se sintetiza la información corológica disponible (área de ocupación) de la especie, se ofrecen datos sobre fenología de los adultos y tipología general de los hábitats que ocupa, se concretan los rasgos diagnósticos y se ilustra la variabilidad morfológica (incluido el patrón cromático) detectada tras el estudio de más de un centenar de ejemplares, conservados en su mayor parte en la colección del Museo Nacional de Ciencias Naturales (MNCN-CSIC, Madrid, España).

\section{Material y metodos}

Esta contribución se basa fundamentalmente en el estudio de un total de 133 ejemplares de $H$. duodecimmaculatus. Una pequeña parte de este material (13 ejemplares) proviene de recientes muestreos efectuados por los autores, durante julio de 2013, en el extremo occidental del Rif (región de Yebala, en la Península Tingitana; véase El Gharbaoui, 1981). Asimismo, se ha examinado la totalidad del material de $H$. duodecimmaculatus conservado en la colección del Museo Nacional de Ciencias Naturales, MadridEspaña (MNCN), 120 ejemplares, colectados en su mayoría por M. Martínez de la Escalera durante sus estancias en Marruecos (véase García-París \& Ruiz, 2011; Martín Albaladejo, 2011; Pina, 2011). La mayor parte de este material se conserva en seco, excepto 5 ejemplares de la población rifeña que se conservan en etanol. La información del material estudiado se detalla en el Apéndice 1. De igual forma, se han incluido datos de material no revisado por los autores (Marco A. Bologna, com. pers. 2014) incluidos en el Apéndice 2.

El estudio morfológico se efectuó sobre ejemplares conservados en seco, mediante observación con estereomicroscopio. La genitalia masculina y la valvífera de la hembra fueron extraídas tanto a ejemplares secos previamente rehidratados como a ejemplares frescos (material rifeño capturado recientemente), y se montaron en cartulinas con resina DMHF, que se pincharon con su respectivo ejemplar. Las medidas se efectuaron con micrómetro acoplado a uno de los oculares y los dibujos con cámara clara. Las fotografías se efectuaron con cámara digital.

\section{Resultados y discusión}

DisTRIBUCIÓN GEOGRÁFICA Y ASPECTOS AUTOECOLÓGICOS

Desde la descripción de $H$. duodecimmaculatus y durante todo el siglo XIX y principios del XX, los 


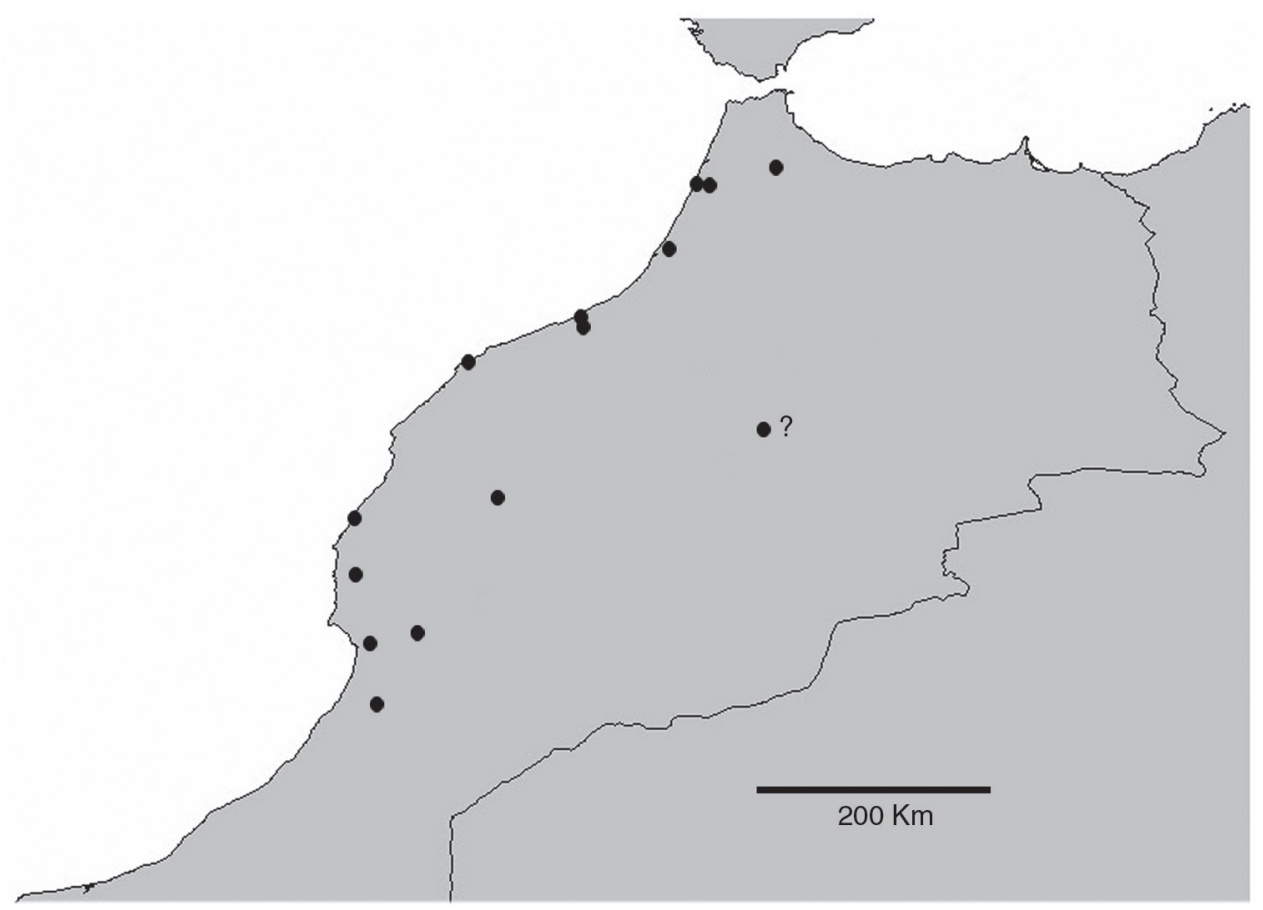

Fig. 1.- Mapa de Marruecos con las localidades conocidas de Hycleus duodecimmaculatus (ver Tabla I). El signo "?" se refiere a la localidad de Tassent (Alto Atlas oriental), que necesita confirmación.

Fig. 1.- Map of Morocco showing known localities of Hycleus duodecimmaculatus (see Table I). Question mark refers to Tassent (Eastern High Atlas), a locality in need of confirmation.

autores que se refieren a ella se limitan a recoger la vaga distribución dada en la descripción original (terra typica), "Barbarie", "Afrique boréal" o "Nordafrika", sin mayor concreción, y la adscriben indistintamente a Mylabris Fabricius, 1775, Decatoma Dejean, 1821, Decapotoma Voigts, 1902 o Zonabris Harold, 1879 (Chevrolat, 1840; Marseul, 1870, 1873; Gemminger, 1870; Beauregard, 1890; Sumakov, 1915; Borchmann, 1917; Mader, 1927).

Martínez de la Escalera (1909, sub Zonabris duodecimmaculata) la señala por primera vez de Marruecos, en concreto de las localidades costeras atlánticas de Casablanca, Mazagán (actual El-Jadida) y Mogador (actual Essaouira). Posteriormente, añade los registros de Larache y Marraquech, sobre material colectado por él mismo (Martínez de la Escalera, 1914). Sumakov (1930, sub Mylabris duodecimmaculata) repite las citas ofrecidas por M. Martínez de la Escalera y más tarde la cita del Bosque de Mamora (Kenitra, 2-V y 20-VI1926), en un alcornocal (Quercus suber L.) sobre arenas, en diversas herbáceas en flor, principalmente Scolymus maculatus L. (Sumakov, 1934). Pardo Alcaide [1954a, sub Mylabris (Gorrizia) duodecimmaculata] le atribuye una distribución ceñida al litoral atlántico, entre Larache al norte y Essaouira al sur. Kocher (1956a) indica la misma distribución y posteriormente añade una localidad más meridional, Ait-Melloul $(12 \mathrm{~km}$ al sureste de Agadir) en la región del Sus (Kocher, 1964), y un registro del Alto Atlas oriental, Tassent, $1800 \mathrm{~m}$ de altitud (Kocher, 1969; "coll. ISC ex Antoine”), 11 km al noroeste de Imilchil, en principio excéntrico al área de extensión de la especie. Por último, Casset \& Faucheux [2012, sub Hycleus (Mesoscutatus) duodecimmaculatus] la señalan de Tamanar, Oued Igouzoulen, 190 m (entre Agadir y Essaouira, tan solo unos $13 \mathrm{~km}$ al interior de la costa) a partir de un individuo hallado el 29-IV-2005 sobre una inflorescencia amarilla de Asteraceae indeterminada. Cabe resaltar que tanto Pardo Alcaide (1954a) como Kocher (1956a) obviaron el registro de Marrakech aportado por M. Martínez de la Escalera, sin comentario alguno al respecto. Por tanto, los registros precisos de $H$. duodecimmaculatus se limitan a nueve (uno de ellos no recogido por autores más modernos y otro, en el Alto Atlas oriental, que necesita confirmación), localizados en su mayor parte en la costa atlántica marroquí (Tabla 1; Fig. 1).

Recientemente hemos localizado una población de esta especie en la vertiente oriental del macizo de Bou-Hachem, Rif occidental (Península Tingitana), en una zona interior de media montaña $(995 \mathrm{~m}$ de altitud), a unos $60 \mathrm{~km}$ al este de la costa atlántica. Este registro es el más nororiental para la especie y el primero del Rif. Por otro lado, entre el material estudiado del MNCN se encuentra un ejemplar de pequeño tamaño procedente de Marrakech (Martínez de la Escalera leg.), confirmando la cita de esta localidad ignorada por autores posteriores, corroborada a su vez por un ejemplar existente en la colección M. Bologna (ver Apéndice 2). Asimismo, añadimos cuatro nuevas localidades: Ouled Mesbah Rissana 
Tabla 1.- Localidades conocidas de Hycleus duodecimmaculatus. Datos de precipitación media anual obtenidos de: (1) Benabid (1982); (2) Le Houerou (1989).

Table 1.- Known localities of Hycleus duodecimmaculatus. Average annual rainfall obtained from: (1) Benabid (1982); (2) Le Houerou (1989).

\begin{tabular}{|c|c|c|c|c|}
\hline Localidad (Cita) & $\begin{array}{c}\text { Región administrativa/ } \\
\text { Provincia }\end{array}$ & $\begin{array}{l}\text { Coordenadas } \\
\text { geográficas }\end{array}$ & Altitud & $\begin{array}{l}\text { Precipitación media } \\
\text { anual (fuente) }\end{array}$ \\
\hline $\begin{array}{l}\text { Larache (Martínez de la } \\
\text { Escalera, 1914) }\end{array}$ & Tánger-Tetouan / Larache & $\begin{array}{l}35^{\circ} 11^{\prime} \mathrm{N} \\
06^{\circ} 08^{\prime} \mathrm{O}\end{array}$ & $0-50 \mathrm{~m}$ & $683 \mathrm{~mm}(1)$ \\
\hline Ouled Mesbah Rissana (inédita) & Tánger-Tetouan / Larache & $\begin{array}{l}35^{\circ} 10^{\prime} \mathrm{N} \\
06^{\circ} 00^{\prime} \mathrm{O}\end{array}$ & $23 \mathrm{~m}$ & $839,5 \mathrm{~mm}(1)$ \\
\hline $\begin{array}{l}\text { Alimadene-Bou Hachem } \\
\text { (inédita) }\end{array}$ & Tánger-Tetouan / Larache & $\begin{array}{l}35^{\circ} 16^{\prime} 19^{\prime \prime} \mathrm{N} \\
5^{\circ} 26^{\prime} 41^{\prime} \mathrm{O}\end{array}$ & $995 \mathrm{~m}$ & $\begin{array}{l}2168 \mathrm{~mm} \text { (1: en Yebel } \\
\text { Bou-Hachem) }\end{array}$ \\
\hline $\begin{array}{l}\text { Bosque Mamora-Kenitra } \\
\quad(\text { Sumakov, 1934) }\end{array}$ & $\begin{array}{l}\text { Gharb-Chrarda-Beni } \\
\text { Hssen / Kenitra }\end{array}$ & $\begin{array}{l}34^{\circ} 12^{\prime} \mathrm{N} \\
06^{\circ} 34^{\prime} \mathrm{O}\end{array}$ & $60-80 \mathrm{~m}$ & $596 \mathrm{~mm}(2)$ \\
\hline $\begin{array}{l}\text { Casablanca (Martínez de la } \\
\text { Escalera, 1909) }\end{array}$ & $\begin{array}{l}\text { Grand Casablanca / } \\
\text { Casablanca }\end{array}$ & $\begin{array}{l}33^{\circ} 32^{\prime} \mathrm{N} \\
07^{\circ} 41^{\prime} \mathrm{O}\end{array}$ & $40-60 \mathrm{~m}$ & $443 \mathrm{~mm}(2)$ \\
\hline Bouskoura (inédita) & $\begin{array}{l}\text { Grand Casablanca / } \\
\text { Casablanca }\end{array}$ & $\begin{array}{l}33^{\circ} 27^{\prime} \mathrm{N} \\
07^{\circ} 38^{\prime} \mathrm{O}\end{array}$ & $135 \mathrm{~m}$ & $\begin{array}{l}443 \mathrm{~mm}(2: \text { en } \\
\text { Casablanca) }\end{array}$ \\
\hline $\begin{array}{l}\text { El Jadida (Martínez de la } \\
\text { Escalera, 1909) }\end{array}$ & Doukkala-Abda / El Jadida & $\begin{array}{l}33^{\circ} 14^{\prime} \mathrm{N} \\
08^{\circ} 30^{\prime} \mathrm{O}\end{array}$ & $0-25 m$ & 366 mm (2) \\
\hline $\begin{array}{l}\text { Essaouira (Martínez de la } \\
\text { Escalera, 1914) }\end{array}$ & $\begin{array}{l}\text { Marraquech-Tensift-Al } \\
\text { Haouz / Essaouira }\end{array}$ & $\begin{array}{l}31^{\circ} 30^{\prime} \mathrm{N} \\
09^{\circ} 45^{\prime} \mathrm{O}\end{array}$ & $0-10 m$ & $295 \mathrm{~mm}(2)$ \\
\hline $\begin{array}{l}\text { Tamanar (Casset \& Faucheaux, } \\
\text { 2012) }\end{array}$ & $\begin{array}{l}\text { Marraquech-Tensift-Al } \\
\text { Haouz / Essaouira }\end{array}$ & $\begin{array}{l}31^{\circ} 00^{\prime} \mathrm{N} \\
09^{\circ} 40^{\prime} \mathrm{O}\end{array}$ & $290-315 \mathrm{~m}$ & $313 \mathrm{~mm}(2)$ \\
\hline $\begin{array}{l}\text { Marraquech (Martínez de la } \\
\text { Escalera, 1914) }\end{array}$ & $\begin{array}{l}\text { Marraquech-Tensift-Al } \\
\text { Haouz / Marraquech }\end{array}$ & $\begin{array}{l}31^{\circ} 37^{\prime} \mathrm{N} \\
07^{\circ} 57^{\prime} \mathrm{O}\end{array}$ & $460-490 \mathrm{~m}$ & $250 \mathrm{~mm}(2)$ \\
\hline Ait Melloul (Kocher, 1964) & $\begin{array}{l}\text { Souss-Massa-Draa / } \\
\text { Inezgane Ait Melloul }\end{array}$ & $\begin{array}{l}30^{\circ} 19^{\prime} \mathrm{N} \\
09^{\circ} 30^{\prime} \mathrm{O}\end{array}$ & $15-25 \mathrm{~m}$ & $225 \mathrm{~mm}(2)$ \\
\hline Taroudant (inédita) & $\begin{array}{l}\text { Souss-Massa-Draa / } \\
\text { Taroudant }\end{array}$ & $\begin{array}{l}30^{\circ} 27^{\prime} \mathrm{N} \\
08^{\circ} 53^{\prime} \mathrm{O}\end{array}$ & $225 \mathrm{~m}$ & $231 \mathrm{~mm}(2)$ \\
\hline Arbaa Ait Ahmed (inédita) & $\begin{array}{l}\text { Souss-Massa-Draa / } \\
\text { Tiznit }\end{array}$ & $\begin{array}{l}29^{\circ} 48^{\prime} \mathrm{N} \\
09^{\circ} 21^{\prime} \mathrm{O}\end{array}$ & $141 \mathrm{~m}$ & 189 mm (2: en Tiznit) \\
\hline Tassent (Kocher, 1969) & Tadla-Azilal / Beni Mellal & $\begin{array}{l}32^{\circ} 14^{\prime} \mathrm{N} \\
05^{\circ} 40^{\prime} \mathrm{O}\end{array}$ & $1800-1900 \mathrm{~m}$ & $\begin{array}{l}300-400 \mathrm{~mm} \\
\text { (2: en Imilchil) }\end{array}$ \\
\hline
\end{tabular}

(=Ulad Mesbah), ubicada a $12 \mathrm{~km}$ al este de Larache a baja altitud, $15 \mathrm{~m}(\mathrm{MNCN})$; Bouskara, muy cercana a Casablanca (sólo $9 \mathrm{~km}$ al sur); Taroudant, al este de Agadir, unos $68 \mathrm{~km}$ al interior, a baja altitud (230 m); y Arbaa Ait Ahmed, región de Tiznit, unos $40 \mathrm{~km}$ al interior de la línea de costa, en el valle del Oued Souss, también a baja altitud $(138 \mathrm{~m})$. Esta última localidad es la más meridional conocida para la especie.

La localidad rifeña, integrada en el Parque Regional de Bou-Hachem (en la región o comarca natural de Yebala), presenta materiales de tipo areniscas (flysch de Beni-Ider) que dan lugar a sustratos ácidos, con un ombrotipo hiperhúmedo debido a una importante influencia atlántica (precipitación media anual $=2168.1 \mathrm{~mm}$ en la estación meteorológica de Yebel Bou-Hachem; ver Benabid, 1982), en el piso bioclimático mesomediterráneo (Benabid, 1982, 1984; Benabid \& Fennane, 1994; Charco, 1999). La vegetación potencial y dominante en estas montañas, a media altitud, está constituida por densos y bien estructurados bosques de alcornocal-quejigal (especies arbóreas dominantes: Quercus suber L. y Quercus canariensis Willd.), con abundante sotobosque de Arbutus unedo L., Erica arborea L. y Cistus populifolius L. El paraje donde se hallaron los individuos de $H$. duodecimmaculatus se corresponde con una zona de cultivo en barbecho, situada en el límite entre el bosque y las amplias áreas deforestadas para explotaciones agrícolas tradicionales (principalmente cerealísticas y olivares) existentes en el piedemonte y cotas bajas de esta región montañosa (véase Taiqui, 1997; Taiqui \& Martín Cantarino, 1997; Taiqui et al., 2008; Chambouleyron, 2012). Los individuos se encontraban activos a partir de las 08:30 h (hora solar), visitando compuestas (Asteraceae) ruderales de inflorescencia amarilla, principalmente Andryala integriflora $\mathrm{L}$.

Los individuos de Bou-Hachem apenas presentaban desgaste tegumentario, lo que es indicativo de una reciente emergencia. Según los escasos datos fenológicos disponibles (bibliográficos y fechas de captura del 

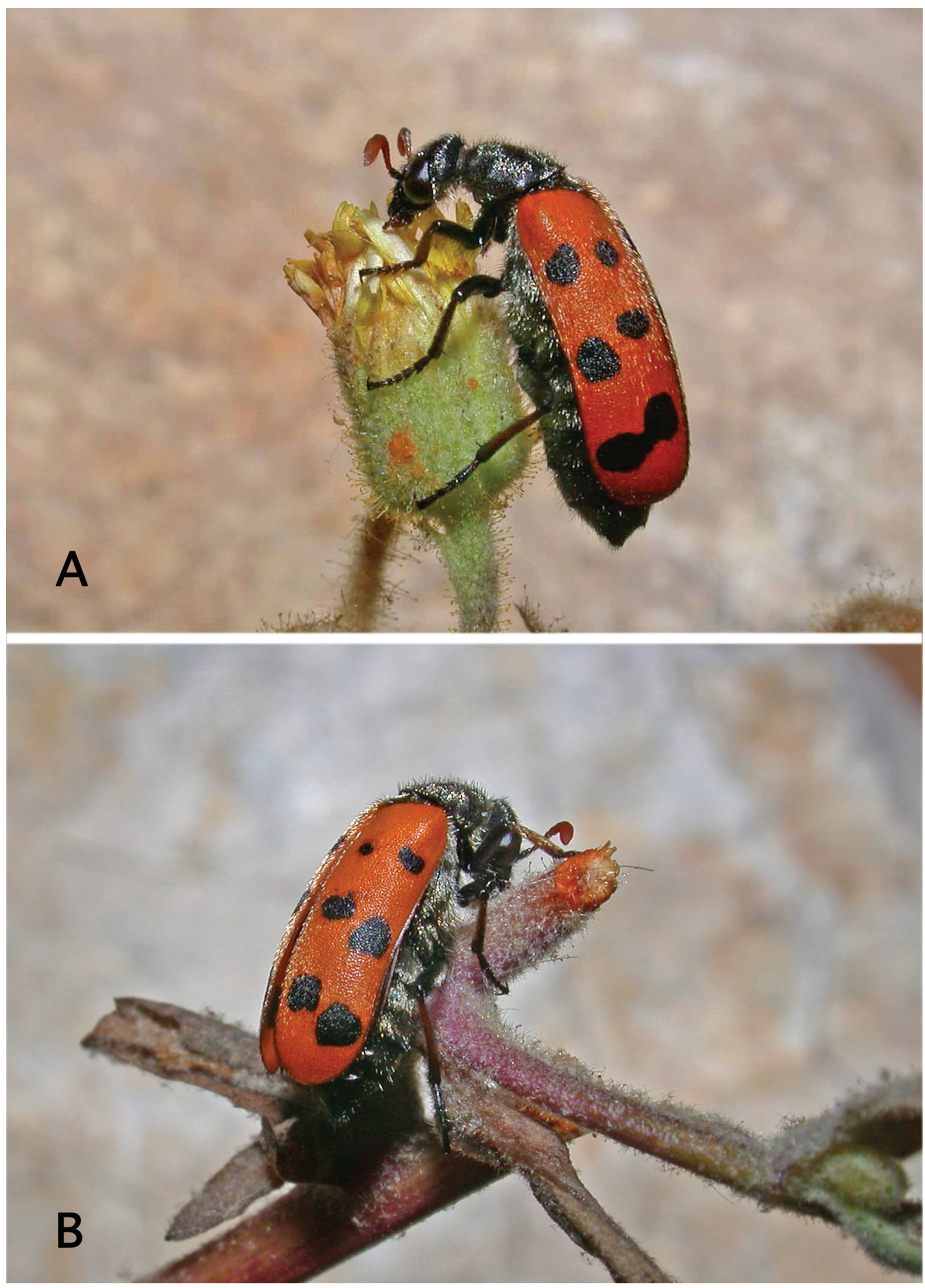

Fig. 2.- Habitus de ejemplares vivos de Hycleus duodecimmaculatus del Rif occidental (Bou-Hachem). Nótese la pilosidad corta dorada de los élitros (sobre todo en la región periescutelar), así como los pelos negros erectos del pronoto mezclados con densa pilosidad argentada (Photograh by M. García-París).

Fig. 2.- Habitus of living specimens of Hycleus duodecimmaculatus from Western Rif (Bou-Hachem). Note the golden elytral pilosity (particularly around the scutellum), and the black erect pronotal hair amongst dense silver pilosity (Photograh by M. García-París). 

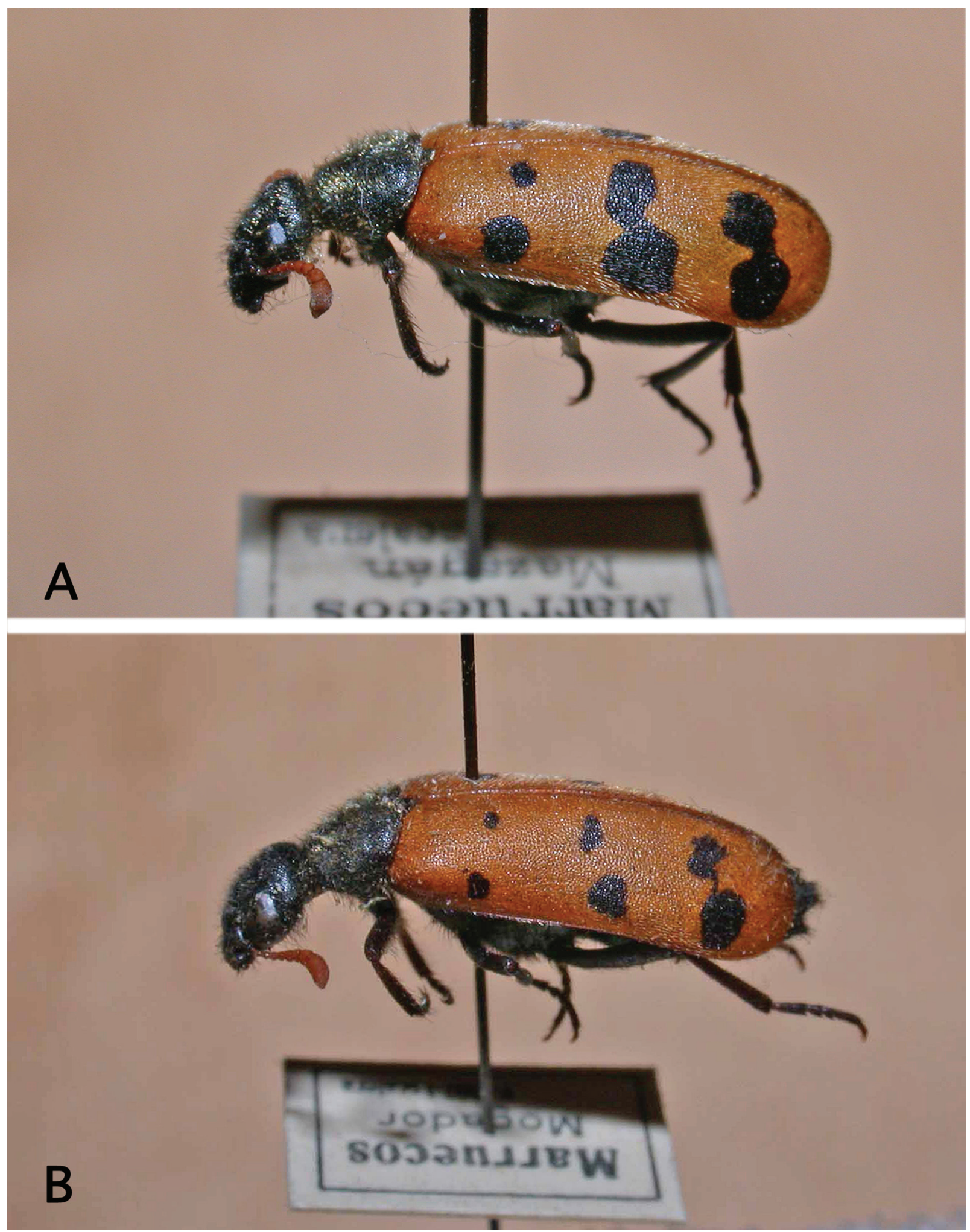

Fig. 3.- Facies de ejemplares de Hycleus duodecimmaculatus de la costa atlántica (Marruecos, MNCN): A) Mazagán (= El Jadida); B) Mogador (= Essaouira). Nótese la pilosidad corta argentada de los élitros en ambos ejemplares.

Fig. 3.- Facies of specimens of Hycleus duodecimmaculatus from the Atlantic Coast (Morocco, MNCN): A) Mazagán (=El Jadida); B) Mogador (= Essaouira). Note the silvery elytral pilosity in both specimens.

material estudiado), los adultos se encuentran activos entre finales de abril y julio en zonas bajas costeras o subcosteras (bosque de Mamora, Tamanar, Essaouira).
En el caso de la población rifeña, el periodo de actividad imaginal sería más tardío que en las poblaciones de baja altitud, haciendo aparición a finales de julio 


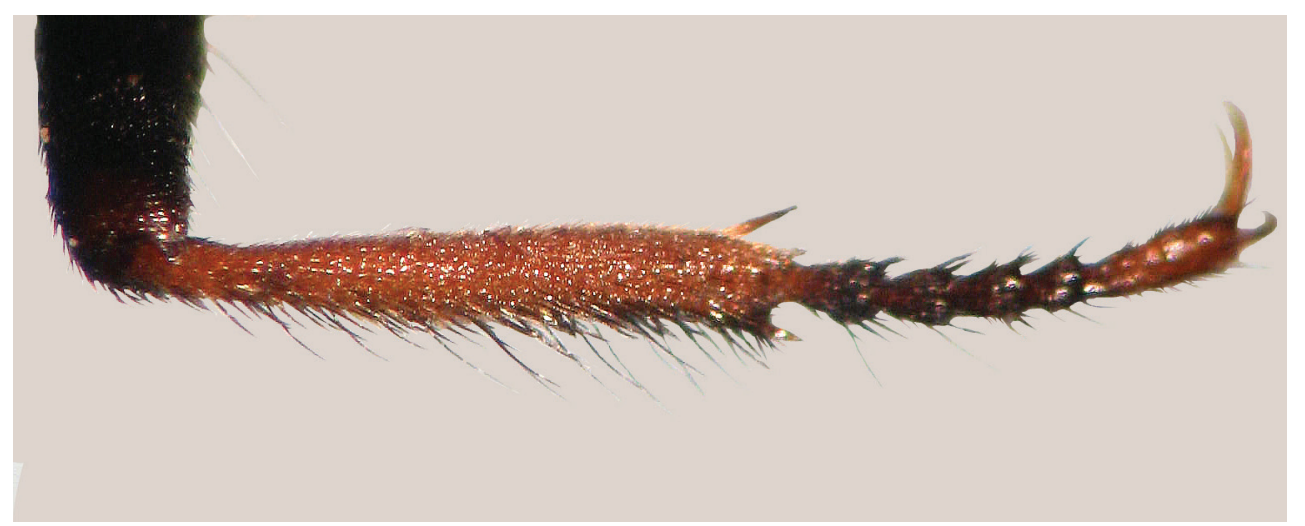

Fig. 4.- Tibia anterior derecha de la hembra de Hycleus duodecimmaculatus (Bou-Hachem, Marruecos). Nótese la larga pilosidad negra en la arista externa (longitud de la tibia: $1.9 \mathrm{~mm}$ ).

Fig. 4.- Right fore-tibia of Hycleus duodecimmaculatus female (Bou-Hachem, Marruecos). Note long black pilosity along the external edge (tibial length: $1.9 \mathrm{~mm}$ ).

y se prolongaría al menos hasta principios de agosto, si bien estaría sujeto a las lógicas variaciones interanuales; a este respecto, el año de hallazgo de este núcleo poblacional fue de temperaturas primaverales moderadas y elevada y persistente pluviometría hasta finales de junio.

En definitiva, según la información corológica disponible (Tabla 1; Fig. 1), el área de distribución de $H$. duodecimmaculatus se extiende por la franja costera-subcostera del Marruecos atlántico, entre Larache al norte y el valle medio del Souss-región de Tiznit, al sur, con penetraciones hacia el interior en zonas favorables: extremo noroccidental del Rif (Bou-Hachem), en terrenos silíceos (ácidos) con alcornocales más o menos abiertos (o sustituidos por cultivos) de clara influencia atlántica; Marrakech, hacia donde posiblemente penetre a favor del valle del Oued Tensift; Taroudant, en el tramo medio-bajo del Oued Souss, y Arbaa Ait Ahemed, en el valle del Oued Massa. Ocupa los pisos bioclimáticos infra-, termomediterráneo $\mathrm{y}$, puntualmente, el mesomediterráneo inferior, en regiones generalmente de relieves suaves, baja altitud y suelos mayoritariamente arenosos, con ombrotipos entre árido-semiárido e hiperhúmedo siguiendo un gradiente N-S de pluviosidad decreciente (ver e.g. Benabid, 1985; Le Houerou, 1989; Benabid \& Fennane, 1994), en la denominada "región atlántica" de Marruecos (e.g. Kocher, 1956b; Benabid \& Fennane, 1994).

Estimamos necesario confirmar la presencia de un núcleo poblacional estable de $H$. duodecimmaculatus en Alto Atlas oriental (Tassent, $1800 \mathrm{~m}$ ), por los siguientes motivos: (1) las condiciones ambientales (media-alta montaña, litologías calcáreas, clima con inviernos muy fríos y marcada continentalidad) y tipología del hábitat [principalmente encinares supramediterráneos abiertos de Quercus rotundifolia Lam. adscribibles a las asociaciones Buxo balearicae-Quercetum rotundifoliae y Ormenido
scariosae-Quercetum rotundifoliae, en zonas con bioclima frío y seco, con presencia en cotas superiores de Cedrus atlantica (Endl.) Carrière; e.g. Quézel et al., 1987; Benabid \& Fennane, 1994; Charco, 1999; Taleb \& Fennane, 2008] son muy diferentes de las del resto del área de ocupación (sensu UICN, 2001) de la especie; (2) se encuentra netamente distanciado del registro más cercano, unos $220 \mathrm{~km}$ al este de Marrakech, y no se conocen poblaciones intermedias en regiones montañosas del Alto Atlas occidental y central relativamente bien prospectadas (e.g. Martínez de la Escalera \& Martínez de la Escalera, 1913; Martínez de la Escalera, 1914; Kocher, 1953, 1956b); en este sentido, en caso de confirmarse su existencia, este hipotético núcleo poblacional se encontraría aparentemente aislado del área de extensión mayoritaria de la especie.

CARACTERES DIAGNÓSTICOS Y VARIABILIDAD MORFOLÓGICA Hycleus duodecimmaculatus se integra en el grupo o "sección" denominada por Pardo Alcaide (1954a: 81) Mesoscutata (especie-tipo: Mylabris duodecimpunctata Olivier, 1811, por designación original), bien caracterizada por presentar el mesosterno con la zona media anterior modificada a modo de escudo mesosternal (scutum central, sensu Pardo Alcaide, 1954a). En la actualidad, Mesoscutata no es considerado subgénero de Hycleus, al igual que las demás secciones propuestas por Pardo Alcaide (1954a, 1955), aunque podrían constituir posibles líneas filéticas o linajes intragenéricos [para una revisión del estado de la taxonomía interna y límites de Hycleus véase Bologna (1991, 1994b, 2008, 2009), Bologna \& Pinto (2002) y Bologna \& Turco (2007)].

En el ámbito de los representantes marroquíes de esta amplia sección (un total de 10 especies), H. duodecimmaculatus se caracteriza sin dificultad por los siguientes rasgos (Fig. 2: aspecto general en vivo): longitud entre $7-12 \mathrm{~mm}$; cabeza, pronoto y región ventral negro brillante; antenas (excepto los dos 

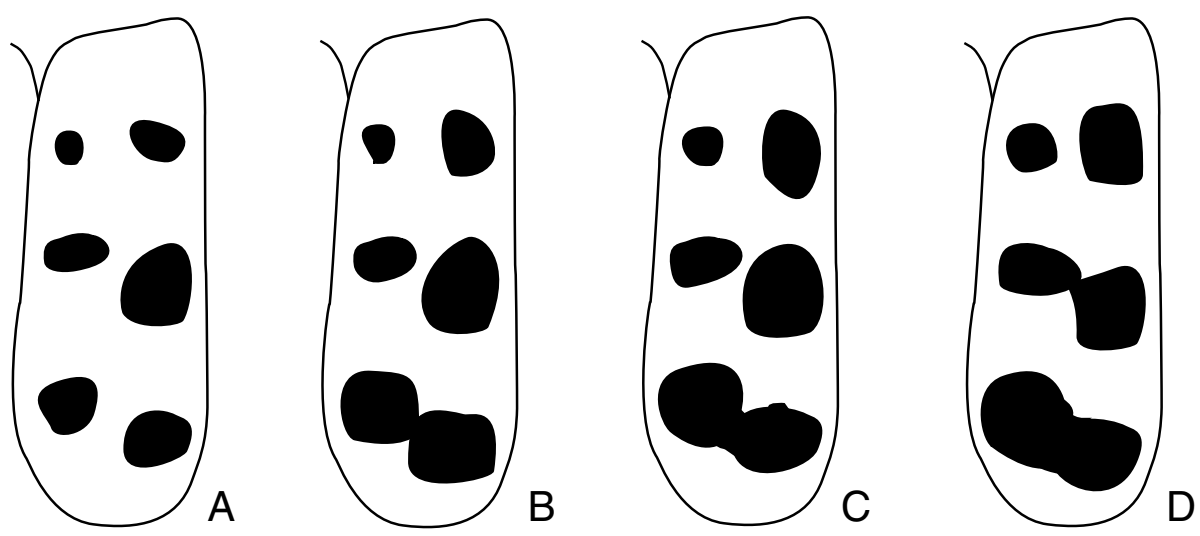

Fig. 5.- Variabilidad en el diseño del élitro derecho de Hycleus duodecimmaculatus. La frecuencia $(n=128)$ de cada diseño es: A: $35.1 \%$; B: $58.6 \%$; C: 5.5\%; D: 0.8\%.

Fig. 5.- Variability of the right elytral pattern of Hycleus duodecimmaculatus. Frequency $(n=128)$ of each pattern is: A: $35.1 \%$; B: $58.6 \%$; C: $5.5 \%$; D: 0.8\%.

primeros artejos), tibias y palpos rojizo-anaranjados, los dos últimos a veces sólo rufescentes, más o menos oscurecidos; antenas de diez artejos aparentes (X y XI fusionados); sutura frontal muy arqueada; ojos anchos y globosos; mandíbulas cortas y de márgenes externos ampliamente curvados; maxilas de los machos con una foseta oval central finamente punteada (característico de la "sección" Androfoveata sensu Pardo Alcaide, 1950, 1954a); sutura mesosternal anterior rectilínea, el escudo central liso y brillante, desprovisto de punteado y pilosidad; pronoto negro, con el tegumento brillante (aunque el denso punteado le imprimen un aspecto general semimate), casi igual de largo que ancho, con punteado fuerte pero fino, denso, con puntos casi contiguos; élitros rojizo-anaranjados, con seis manchas redondeadas negras, distribuidas por pares (anterior, medio y posterior), las de un mismo par a veces fusionadas; pubescencia del cuerpo en su mayor parte argentada (en las poblaciones costeras y subcosteras) y machos sin largas sedas en la arista externa de las tibias anteriores, que sin embargo aparecen en las hembras (en parte Pardo Alcaide, 1954a; obs. pers.).

Marseul (1870: 210-211; 1873: 570-571), Martínez de la Escalera (1909: 247) y Sumakov (1915: 64) indicaron erróneamente, en sus respectivas claves de determinación, que presenta las patas negras, y el segundo autor además señala que los palpos también son negros, siendo realmente las tibias entre rojizoamarillentas y castaño-rojizas más o menos oscurecidas (nunca totalmente negras) y los palpos maxilares y labiales con los artejos basales negros o castaño muy oscuros y el artejo distal de color rojizo-amarillento en la totalidad de los ejemplares estudiados, y en ocasiones esta coloración clara a veces se extiende difusamente al artejo predistal en los palpos maxilares.

La pilosidad pronotal es doble, constituida por abundantes pelos argentados, (a veces con reflejos amarillento-dorados: población de Bou-Hachem), aplicados contra el tegumento y otros negros, erectos, más escasos y dispersos (carácter no indicado por Pardo Alcaide, 1954a), siendo muy variable la cantidad de estos pelos negros pronotales, que además se desprenden fácilmente con el roce. Pardo Alcaide (1954a, 1954b) puso de manifiesto la elevada variabilidad inter- e intrapoblacional en el color de la pilosidad (especialmente del pronoto y élitros) en las especies de Hycleus marroquíes, sobre todo las adscritas a la "sección" Mesoscutata, y que en ocasiones determinados patrones de coloración se encuentran fijados en poblaciones bien localizadas geográficamente. Un ejemplo sería el caso de H. igneopubescens Martínez de la Escalera, 1909, de la región del Souss (= Sus, suroeste de Marruecos), caracterizada por presentar los pelos de los élitros mayoritariamente dorados (Pardo Alcaide, 1954a; García-París \& Ruiz, 2011), que ha sido sucesivamente tratada como variedad de $H$. ruficornis por Pardo Alcaide (1954a), como subespecie por Kocher (1956a) y actualmente considerada sinónimo de ésta (Bologna, 2008).

Según Pardo Alcaide (1954a: 82) la pilosidad corporal y elitral de $H$. duodecimmaculatus es argentada, lo que coincide con la observada en los élitros de los ejemplares de las poblaciones costeras y subcosteras atlánticas examinados (Fig. 3). Sin embargo, en el caso de los individuos de la población del Rif occidental (Bou-Hachem; longitud: 10.1$11.7 \mathrm{~mm}$; media = 11.3; dentro del rango de tamaño indicado por Pardo Alcaide, 1954a), la pilosidad elitral es dorada en lugar de plateada (Fig. 2) con escasos pelos negros, tumbados y dispersos, algo más abundantes en el tercio basal (de forma análoga al mencionado $H$. igneopubescens); en el ejemplar de Marrakech los pelos elitrales son blanquecinoamarillentos, semidorados. Por lo tanto, la pilosidad de los élitros de $H$. duodecimmaculatus varía 


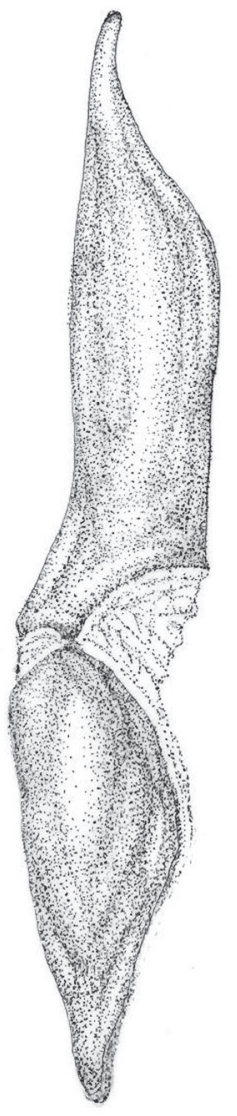

A

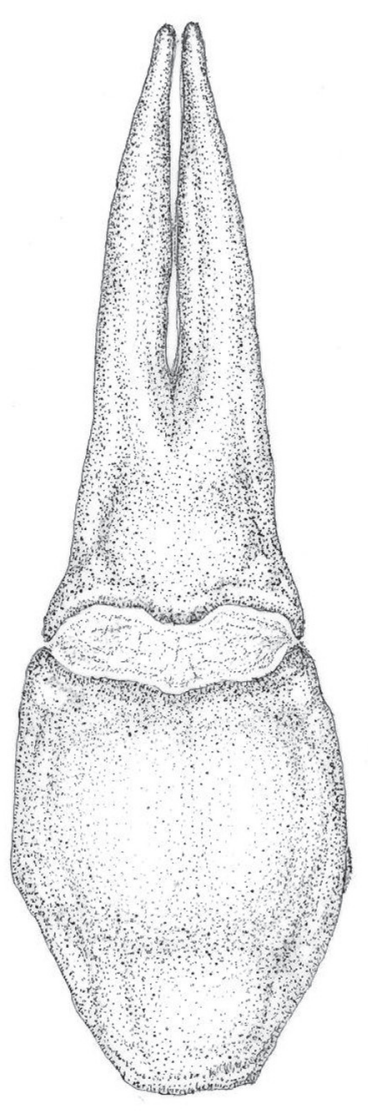

B

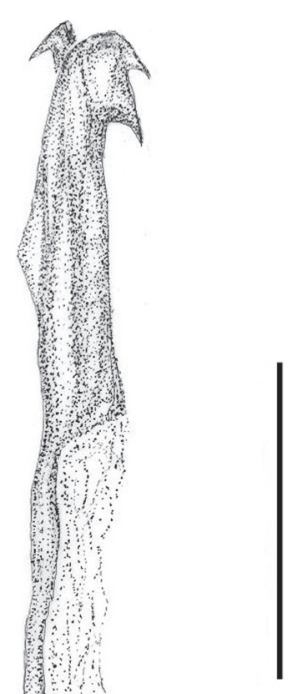

C

Fig. 6.- Genitalia masculina de Hycleus duodecimmaculatus (Bou-Hachem, Marruecos); A.- tegmen en visión lateral; B.- tegmen en visión dorsal; C.- lóbulo medio en visión lateral. Escala: 0.5 mm (J.L. Ruiz del.).

Fig. 6.- Male genitalia of Hycleus duodecimmaculatus (Bou-Hachem, Marruecos); A.- tegmen in lateral view; B.- tegmen in dorsal view; C.- median lobe in lateral view. Scale bar: $0.5 \mathrm{~mm}$ (J.L. Ruiz del.).

entre totalmente plateada y dorada con algunos pelos negros, aunque parece ser estable a nivel intrapoblacional.

El punteado del pronoto es fino pero muy denso y bien impreso, subcontiguo y en general uniformemente repartido, excepto en una estrecha y corta depresión centro-longitudinal, a modo de pequeña incisión central con muy ligera variación en su longitud. La estructura mesosternal es muy homogénea en conjunto, con leves variaciones en su anchura y longitud, así como en la amplitud del escudo central. Por otro parte, en algunos ejemplares se observa en las antenas una traza de sutura entre el artejo $\mathrm{X}$ y el XI, más aparente en la región ventral, que en ocasiones se intuye como un leve estrechamiento de límites difusos, si bien en la mayoría de los individuos es inexistente esta traza de sutura, presentando sendos antenómeros totalmente fusionados. La variación en el color de las antenas es muy estrecha, restringida a una tonalidad más o menos rojiza o amarillenta, pero en general los artejos III a VI son más oscuros y del VII al X (constituido por el X y XI fusionados) se hacen progresivamente más claros (amarillento-anaranjado).

Como carácter dimórfico sexual no señalado anteriormente, se ha observado que las hembras de $H$. duodecimmaculatus, al contrario que los machos, presentan largas sedas negras semierectas en el borde externo de las tibias anteriores, además de numerosos pelos negros cortos y tumbados, estos últimos ligeramente más gruesos y algo más largos que los pelos amarillento-plateados que cubren la totalidad de la tibia (Fig. 4); asimismo, los dos primeros artejos del protarso de la hembra portan algunos escasos pelos negros alargados en su ápice externo, similares a los de las protibias, y ausentes en los machos. Este último rasgo ha sido señalado por Pardo Alcaide $(1950,1954 a)$ como característico de los machos (nunca de las hembras) de algunas especies del grupo de H. polymorphus (Pallas, 1771), al que este autor denominó "sección" Androtarsata [nombre disponible a partir de Pardo Alcaide (1954a), véase García-París et al. (2010)]. 


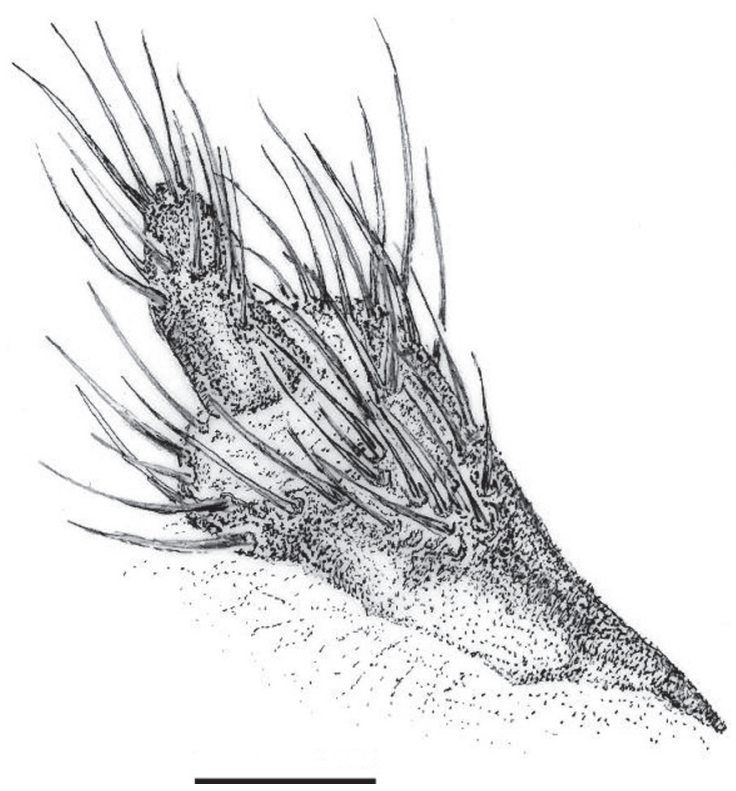

Fig. 7.- Valvífera y estilo de la hembra de Hycleus duodecimmaculatus (Bou-Hachem, Marruecos). Escala: $0.1 \mathrm{~mm}$ (J.L. Ruiz del.).

Fig. 7.- Female valvifer and style of Hycleus duodecimmaculatus (Bou-Hachem, Marruecos). Scale bar: $0.1 \mathrm{~mm}$ (J.L. Ruiz del.).

Como es norma en las especies del género (Bologna, 1991), las hembras muestran además el último esternito abdominal visible apenas o nada escotado, mientras que en los machos esta escotadura es profunda y aparente.

La variabilidad del patrón cromático elitral es relativamente escasa en comparación con otras especies de Hycleus mediterráneo occidentales (ver e.g. Bologna, 1991; Trotta-Moreu \& García-París, 2001) y se refiere fundamentalmente a la fusión, en mayor o menor grado, de las manchas negras semicirculares de la misma serie (Pardo Alcaide, 1954a; obs. pers.), así como al diámetro, transversalidad y sinuosidad de tales manchas. No se han observado fusiones entre manchas de series paralelas, ni tampoco se ha observado reducción extrema o desaparición de alguna de ellas. En la figura 5 se muestran los cuatro patrones en los que se encuadraría la variabilidad de diseño elitral detectado y sus respectivas frecuencias $(\mathrm{n}=128)$. El diseño más frecuente es el B (58.6\%), en el que se fusionan en escaso grado las dos manchas de la serie posterior, seguido del A (35.1\%), en el que no se observa unión alguna entre manchas de ninguna de las series; los patrones C $(5.5 \%)$ y D $(0.8 \%)$ son, con diferencia, los menos frecuentes. En general, la variabilidad en el diseño no parece guardar estrecha relación con la procedencia geográfica de los individuos, si bien en todos los ejemplares examinados de la población de Bou-Hachem, las manchas negras de la serie posterior se encuentran más o menos fusionadas (patrón $\mathrm{B}$, y en tres individuos el C; ver Fig. 5), que por otra parte es el diseño más frecuente en la especie.
La armadura genital masculina es morfológicamente muy constante, con escasa variabilidad en la longitud y anchura de la vaina parameral y lóbulos distales, así como de la falobase. En la figura 6 se ilustra la genitalia masculina de un ejemplar de BouHachem, que respecto a la figurada por Pardo Alcaide (1954a: 83, fig. 40b; este autor no indica la procedencia del material ilustrado) presenta los parámeros con los lóbulos distales más alargados y el diente apical ventral del lóbulo medio más marcado y un poco más cercano al ápice. La valvífera de la hembra (Fig. 7), nunca antes figurada, es estrecha y escasamente esclerotizada, sobre todo en su región inferior, con puntos pilíferos en la mitad dorsal de su cara externa; el estilo es ancho y corto, con pilosidad abundante negra y alargada.

\section{Agradecimientos}

A Marco A. Bologna (Universitá Roma Tre, Roma, Italia) por los datos geográficos enviados y la evaluación crítica del manuscrito, A Mercedes París (MNCN, Madrid, España) por su ayuda en la revisión de la colección del MNCN. Al Instituto de Estudios Ceutíes (Ciudad Autónoma de Ceuta, España) por la ayuda prestada. Este trabajo ha contado con el apoyo del proyecto CGL2010-15786 (Ministerio de Ciencia e Innovación, España).

\section{Referencias}

Beauregard, H., 1890. Les insectes vésicants. Félix Alcan. Paris. xvi +544 pp, 34 láms.

Benabid, A., 1982. Etudes phytoécologique, biogéographique et dinamique des associations et séries sylvatiques du Rif occidental (Maroc). Thèse Doct. ès-Sc. Faculté des Sciences et Techniques St. Jerôme. Université de Droit, d'Economie et des Sciences d'Aix-Marseille. iii+199 pp.

Benabid, A., 1984. Étude phytoécologique des peuplements forestiers et préforestiers du Rif centro-occidental (Maroc). Travaux de l'Institut Scientifique, Rabat, Série Botanique, 34: 1-64, 25 tab., 2 cart.

Benabid, A., 1985. Les écosystèmes forestiers, préforestiers et presteppiques du Maroc: diversité, répartition biogéographique et problèmes posés par leur aménagement. Forêt méditerranéenne, 7(1): 53-64.

Benabid, A. \& Fennane, M., 1994. Connaissances sur la végétation du Maroc: Phytogéographie, phytosociologie et séries de végétation. Lazaroa, 14: 21-97.

Bologna, M. A., 1991. Fauna d'Italia. XXVIII. Coleoptera Meloidae. Calderini. Bologna. xiv +541 pp.

Bologna, M. A., 1994a. Meloidae from Canary and other macaronesian islands (Coleoptera). Miscel-lània Zoològica, [1992], 16: 73-80.

Bologna, M. A., 1994b. I Meloidae della Grecia (Coleoptera). Fragmenta Entomologica, 25(Supplemento): 1-119.

Bologna, M. A., 2008. Meloidae. En: I. Löbl \& A. Smetana (eds.). Catalogue of Palaearctic Coleoptera. Vol. 5. Tenebrionoidea. Apollo Books. Stenstrup. Pp. 370-412. 
Bologna, M. A., 2009. The Meloidae (Coleoptera) of Libya: an annotated catalogue and description of three new species. Annales de la société entomologique de France (n.s.), 45(3): 345-364. http://dx.doi.org/10.1080/003792 71.2009.10697620.

Bologna, M. A. \& Pinto, J. D., 2002. The Old World genera of Meloidae (Coleoptera): a key and synopsis. Journal of Natural History, 36(17): 2013-2102. http://dx.doi. org/10.1080/00222930110062318.

Bologna, M. A. \& Turco, F., 2007. The Meloidae (Coleoptera) of the United Arab Emirates with an updated Arabian checklist. Zootaxa, 1625: 1-33.

Borchmann, F., 1917. Pars 69. Meloidae, Cephaloidae. In: E. Schenkling (ed.). Coleopterorum Catalogus auspiciis et auxilio W. Junk. Junk. Berlin. 208 pp.

Casset, L. \& Faucheaux, M. J., 2012. Quelques Meloidae (Coleoptera) du Maroc atlantique: 1ère liste commentée. Bulletin de la Société des sciences naturelles de l'Ouest de la France, 34(3): 140-147.

Chambouleyron, M., 2012. Contribution à la connaissance de la flore de la péninsule tingitane (Maroc). Lagascalia, 32: 35-227.

Charco, J., 1999. El bosque mediterráneo en el norte de África. Biodiversidad y lucha contra la desertificación. Agencia Española de Cooperación Internacional. Madrid. 370 pp.

Chevrolat, L. A. A., 1840. Description de Mylabrides de Barbarie. Revue Entomologique (G. Silbermann), [1837], 5: 266-279, 1 lám.

El Gharbaoui, A., 1981. La terre et l'homme dans la péninsule tingitane. Etude sur l'homme et le milieu naturel dans le Rif occidental. Travaux de l'Institut Scientifique, Rabat, Série Géologie et Géographie Physique, 15: 1-439, 4 láms., 8 mapas.

García-París, M. \& Ruiz, J. L., 2011. Capítulo 9. Las cantáridas y aceiteras (Coleoptera: Meloidae) en la obra de Manuel Martínez de la Escalera. In: C. Martín Albaladejo \& I. Izquierdo Moya (eds.). Al encuentro del naturalista Manuel Martínez de la Escalera (1867-1949). Monografías del Museo Nacional de Ciencias Naturales. Consejo Superior de Investigaciones Científicas. Madrid: 173-205, láms. 9-14.

García-París, M., Ruiz, J. L. \& Alonso-Zarazaga, M.A., 2010. Catálogo sinonímico de los taxones ibero-baleares de la familia Meloidae (Coleoptera). Graellsia, 66(2): 165-212. http://dx.doi.org/10.3989/graellsia.2010.v66.018.

Gemminger, M., 1870. Meloidae. In: M. Gemminger \& B. Harold. Catalogus Coleopterorum hucusque descriptorum synonymicus et systematicus. VII. Gummi. Monachii: $1801-2180$.

Kocher, L., 1953. Localisations nouvelles ou intéressantes de Coléoptères marocains. Travaux de l'Institute Scientifique Chérifien, série Zoologie, 7: 1-142.

Kocher, L., 1956a. Catalogue commenté des Coléoptères du Maroc. V. Héteromères (Tenebrionides excepts). Travaux de l'Institut Scientifique Chérifien (série Zoologie), 10: 1-107.

Kocher, L., 1956b. Catalogue commenté des Coléoptères du Maroc. Préface-Introduction-Carte. Travaux de l'Institut Scientifique Chérifien (série Zoologie), 7: 1-26, 1 mapa.
Kocher, L., 1964. Catalogue commenté des Coléoptères du Maroc. X. Addenda et Corrigenda. Travaux de l'Institut Scientifique Chérifien (série Zoologie), 30: 1-200.

Kocher, L., 1969. Catalogue commenté des Coléoptères du Maroc. X bis. Nouveaux addenda et corrigenda. Travaux de l'Institut Scientifique Chérifien et de la Faculte des Sciences (série Zoologie), 34: 1-132.

Le Houerou, H. N., 1989. Classification écoclimatique des zones arides (s.1.) de l'Afrique du Nord. Ecologia mediterranea, 15(3/4): 95-144, 7 tabs.

Machado, A. \& Oromí, P., 2000. Elenco de los Coleópteros de las Islas Canarias. Instituto de Estudios Canarios, Monografía 70. La Laguna. 306 pp.

Mader, L., 1927. Meloidae. In: A. Winkler (ed.). Catalogus Coleopterorum regionis palaearcticae, vol. II. Winkler. Wien: 851-888.

Marseul, S. A. de, 1870. Monographie des Mylabrides d'Europe et des contrées limitrophes en Afrique et en Asie. L'Abeille, 7(2e. partie): 1-204.

Marseul, S. A. de, 1873. Monographie des mylabrides. Mémoires de la Société Royale des Sciences de Liège (2), 3: 363-662, 6 láms.

Martín Albaladejo, C., 2011. Capítulo 11. Martínez de la Escalera en el noroeste de África: la huella de sus exploraciones entomológicas. In: C. Martín Albaladejo \& I. Izquierdo Moya (eds.). Al encuentro del naturalista Manuel Martínez de la Escalera (1867-1949). Monografías del Museo Nacional de Ciencias Naturales. Consejo Superior de Investigaciones Científicas. Madrid: 221-241.

Martínez de la Escalera, F. \& Martínez de la Escalera, M., 1913. Una campaña entomológica en el Sus y descripción de los coleópteros recogidos en ella. Trabajos del Museo Nacional de Ciencias Naturales, Serie Zoológica, 8: 1-56.

Martínez de la Escalera, M., 1909. Especies de Zonabris del SW. de Marruecos. Boletín de la Real Sociedad española de Historia Natural, 9: 244-249.

Martínez de la Escalera, M., 1914. Los Coleópteros de Marruecos. Trabajos del Museo Nacional de Ciencias Naturales, serie Zoológica, 11: 1-553.

Olivier, A. G. 1811. Encyclopédie méthodique, ou par ordre de matières; par une société de gens de lettres, de savans et d'artistes; précédé d'un vocabulaire universel servant de table pour tout l'ouvrage, ornée des portraits de Mm. Diderot et d'Alembert, premiers éditeurs de l'Encyclopédie. Histoire naturelle. Insectes. Tome huitième. H. Agasse. Paris. 722 pp.

Pardo Alcaide, A., 1950. Estudios sobre Meloidae II. Los Mylabrini de la Península Ibérica. Boletín de Patología Vegetal y Entomología Agrícola, [1949], 17: 61-82.

Pardo Alcaide, A., 1954a. Études sur les Meloidae. V. Les Mylabrini du Maroc et du Sahara occidental espagnol (Col. Meloidae). Bulletin de la Société des Sciences Naturelles et Physiques du Maroc, 34: 55-88.

Pardo Alcaide, A., 1954b. Estudios sobre Meloidae. IV. Sobre algunos Mylabris Fab. del occidente norteafricano. (Primera parte). Eos, 30: 331-344.

Pardo Alcaide, A., 1955. Estudios sobre Meloidae. VI. Sobre algunos Mylabris (s. lat.) de la región etiópica. 
Institut Royal des Sciences Naturelles de Belgique, 31(49): 1-32.

Pina, J., 2011. Capítulo 19. Manuel y Fernando Martínez de la Escalera por el noroeste de África: expediciones entre 1905 y 1912. In: C. Martín Albaladejo \& I. Izquierdo Moya (eds.). Al encuentro del naturalista Manuel Martínez de la Escalera (1867-1949). Monografías del Museo Nacional de Ciencias Naturales. Consejo Superior de Investigaciones Científicas. Madrid: 365-391.

Quézel, P., Barbero, M. \& Benabid, A., 1987. Contribution à l'étude des groupements forestierset pré-forestiers du Haut Atlas Oriental (Maroc). Ecologia Mediterranea, 13(1/2): 107-117.

Ruiz, J. L., 2000. El estatus taxonómico de Mylabris (Mesosulcata) thamii Kocher, 1963, un endemismo del sur de Marruecos (Coleoptera, Meloidae). Graellsia, 56: 97-106. http://dx.doi.org/10.3989/graellsia.2000.v56.i0.314.

Ruiz, J. L., 2004. Distribución y morfología de Hycleus rungsi (Peyerimhoff, 1935), un endemismo de Maruecos meridional (Coleoptera, Meloidae). Boletín de la Sociedad Entomológica Aragonesa, 34: 95-100.

Ruiz, J. L. \& García-París, M., 2008. Descripción de una nueva especie de Mylabris Fabricius, 1775 del subgénero Ammabris Kuzin, 1954 de Marruecos y redescripción de Mylabris (Ammabris) boghariensis Raffray, 1873 (Coleoptera, Meloidae). Graellsia, 64(1): 107133. http://dx.doi.org/10.3989/graellsia.2008.v64.i1.57.

Sumakov, G. G., 1915. Les espèces paléarctiques du genre Mylabris Fabr. (Coleoptera, Meloïdae). Horae Societatis Entomologicae Rossicae, 42(1): 1-73.

Sumakov, G. G., 1930. Catalogue des espèces paléarctiques de tribu Mylabrina (Coleoptera, Meloidae). Tartu
Ülikooli juures oleva Loodusuurijate Seltsi Aruanded, 37(1-2): 1-114.

Sumakov, G. G., 1934. Inventa Entomologica itineris Hispanici et Maroccani, quod a. 1926 fecerunt Harald et Hakan Lindberg. XXI. Meloides du Bassin Mediterranéen et du Maroc. Commentationes Biologicae, Societas Scientarum Fennica, 4(13): 1-6.

Taiqui, L., 1997. La dégradation écologique au Rif marocain: nécessités d'une nouvelle approach. Mediterranea, Serie de estudios biológicos, 16: 5-17.

Taiqui, L., Martín, J. \& Seva, E., 2008. Structure, diversité et dynamique des subéraies du paysage rural de Chefchaouen. Annales de la recherche forestière du Maroc, 39: 43-57.

Taiqui, L. \& Martín Cantarino, C., 1997. Eléments historiques d'analyse écologiques des paysages montagneux du Rif occidental (Maroc). Mediterranea, Serie de estudios biológicos, 16: 23-35.

Taleb, M. S. \& Fennane, M., 2008. Diversité floristique du Parc National du Haut Atlas Oriental et des Massifs Ayachi et Maâsker (Maroc). Acta Botanica Malacitana, 33: $125-145$.

Trotta-Moreu, N. \& García-París, M., 2001. Los Hycleus Latreille, 1829 (Coleoptera, Meloidae) de la Península Ibérica: distribución geográfica y variabilidad elitral. Graellsia, 57(1): 99-111. http://dx.doi.org/10.3989/ graellsia.2001.v57.i1.298.

UICN (Unión Internacional para la Conservación de la Naturaleza), 2001. Categorías y Criterios de las Listas Rojas de la UICN: Versión 3.1. Comisión de Supervivencia de Especies de la UICN. UICN, Gland, Suiza, Cambridge, Reino Unido. ii +33 pp. 
Apéndice 1.- Material examinado de Hycleus duodecimmaculatus (Olivier, 1811). Las siglas utilizadas son las siguientes: MNCN: Museo Nacional de Ciencias Naturales-CSIC, Madrid (España); JLR: Colección J.L. Ruiz, Ceuta (España).

Appendix 1.- Material examined of Hycleus duodecimmaculatus (Olivier, 1811). The acronyms used are as follows: MNCN: Museo Nacional de Ciencias Naturales-CSIC, Madrid (Spain); JLR: J.L. Ruiz' collection, Ceuta (Spain).

MARRUECOS: 9 ejemplares: "Marruecos, Mazagán, Escalera" $(\mathrm{MNCN}) ; 51$ ejemplares: "Marruecos, Mazagán, M. Escalera" $(\mathrm{MNCN}) ; 14$ ejemplares: "Marruecos, Mogador, M. Escalera" (MNCN); 12 ejemplares: "Marruecos, Mogador, V-1907, Escalera" (MNCN); 5 ejemplares: "Marruecos. Casablanca. Escalera" (MNCN); 1 ejemplar: "1904, Larache" (MNCN); 1 ejemplar: "Ulad Mesbah, VII-1923, J. Gil” (MNCN); 1 ejemplar:
"Marrákes" (MNCN); 1 ejemplar etiquetado y a continuación 14 ejemplares sin etiquetar en la misma serie: "Casablanca" (MNCN); 1 ejemplar etiquetado y a continuación 10 ejemplares sin etiquetar en la misma serie: "Marruecos, Larache, Escalera" (MNCN); 13 ejemplares: "Alimadene, Crtra. Al Hamra-Mulay Abdeselam, Bou-Hachem, Rif occidental, Marruecos, $35^{\circ} 16^{\prime} 19^{\prime \prime} \mathrm{N} 5^{\circ} 26^{\prime} 41^{\prime \prime} \mathrm{O}$, 995 m, 28-VII-2013, M. Ga-París y J.L. Ruiz leg." (MNCN y JLR).

Apéndice 2.- Datos enviados por M. A. Bologna (com. pers., 2014), Universitá Roma Tre (Roma, Italia).

Appendix 2.- Data sent by M. A. Bologna (pers. com., 2014), Universitá Roma Tre (Rome, Italy).

MARRUECOS: 1 ejemplar: "Mogador, 20.7.1959 Ecklein"; 1 ejemplar: "Maroc, Taroudant, bivio [=junction] Aouhouz, 24.vi.91, G. Sama leg."; 1 ejemplar: "Marrakech 16.7.73" [ex Olivella coll.]; 1 ejemplar: "Bouskoura, 17.6.1972, Olivella leg.";
5 ejemplares: "Marocco, Souss-Massa-Drâa, 1 km W/NW centre Commune Arbaa Ait Ahmed, 7.vi.2013, N 2948'12"-W9²1'52", m 141, leg. C. Massarone". 